\title{
Brain imaging correlates of cognitive impairment in depression
}

\author{
Emma J.Thomas and Rebecca Elliott* \\ Neuroscience and Psychiatry Unit, University of Manchester, Manchester, UK
}

\section{Edited by:}

Vince D. Calhoun, University of New

Mexico, USA

Reviewed by:

Julia Stephen, University of New

Mexico Health Sciences Center, USA

Robert J. Thoma, University of New

Mexico, USA

*Correspondence:

Rebecca Elliott, Neuroscience and

Psychiatry Unit, University of

Manchester, Room G907 Stopford

Building, Oxford Road, Manchester

M13 9PT, UK

e-mail: rebecca.elliott@manchester.

ac.uk
This review briefly summarises recent research on the neural basis of cognition in depression. Two broad areas are covered: emotional and non-emotional processing. We consider how research findings support models of depression based on disrupted cortico-limbic circuitry, and how modern connectivity analysis techniques can be used to test such models explicitly. Finally we discuss clinical implications of cognitive imaging in depression, and specifically the possible role for these techniques in diagnosis and treatment planning.

Keywords: depression, cognition, emotion, fMRI, antidepressants, connectivity

\section{INTRODUCTION}

Cognitive deficits, such as reduced concentration and poor memory, are hallmark features of depression listed among diagnostic symptoms (DSM-IV, American Psychiatric Association, 1994). Neuroimaging techniques provide a means to investigate neurobiological abnormalities associated with depression in vivo. Functional neuroimaging can identify brain areas which respond atypically to cognitive challenges, and functional magnetic resonance imaging (fMRI) is now widely used to investigate the neuronal basis of cognitive deficits in depression. Some studies have focused on cognitive challenges based on classic neuropsychological domains (e.g., executive function, memory). Others have explored cognitive mechanisms of emotional disturbance (e.g., negative bias, response to failure). Although results have not been entirely consistent, observed patterns have led to the development of neurobiological models of depression based on functionally abnormal networks. New techniques and approaches are emerging to advance our understanding of disrupted connectivity underpinning cognitive deficits in depression. Relationships between clinical features of depression and the neural basis of cognitive deficits are also being explored. Understanding these relationships has implications for diagnosis and individually tailored treatment strategies. Neuroimaging correlates of cognitive deficits may be an important biomarker for subtypes of depression that respond to different treatment approaches. In this review, we will briefly discuss recent findings with cognitive activation paradigms, before considering how newer techniques and approaches may take us forward, conceptually and clinically.

\section{COGNITIVE ACTIVATION STUDIES IN DEPRESSION}

Haldane and Frangou (2006) reviewed functional neuroimaging studies in mood disorders and concluded that most report associations between depression and increased activity in limbic regions. However, changes in cortical regions are less consistently observed and depend on the experimental paradigm used. This view is supported by another meta-analysis (Fitzgerald et al., 2006), focusing specifically on studies reporting dorsolateral prefrontal cortex (DLPFC) abnormalities. They concluded that while abnormalities were reported relatively consistently, the direction and laterality of observed changes varied depending on the challenge used. Broadly speaking, cognitive challenges used in functional neuroimaging of depression can be divided into non-emotional challenges and emotional challenges, requiring either overt or covert affective processing.

\section{NON-EMOTIONAL COGNITIVE NEUROIMAGING IN CURRENT DEPRESSION \\ Executive function and working memory}

Relatively few studies have examined the neural substrates of nonemotional cognitive deficits in unipolar depression. These have typically focused on executive tasks that depend on functional integrity of the prefrontal cortices. Elliott et al. (1997a) used positron emission tomography to compare neural activity in controls and depressed participants performing a complex planning task. They found that while the groups showed similar overall patterns of activity, this was globally attenuated in depressed subjects, particularly in caudate, thalamus, anterior cingulate (ACC), DLPFC and ventrolateral prefrontal cortex (VLPFC). Task performance in the depressed group was also impaired, suggesting that these attenuations may have reflected poorer performance.

By contrast, Fitzgerald et al. (2008) used a similar planning task with fMRI and reported that depressed participants showed increased activity in regions including right VLPFC, DLPFC and angular gyrus/cuneus. Unlike patients in the Elliott et al. (1997a) study, their performance accuracy was normal. This discrepancy may explain the activation differences, with Fitzgerald et al.s 
patients recruiting additional neuronal resources to achieve normal performance accuracy. Fitzgerald et al. (2008) also demonstrated increased cortical activity in a depressed group using an n-back working memory task where patients' performance was intact. Similarly, Harvey et al. (2005) found increased lateral prefrontal and ACC responses in depressed participants performing an n-back task, in the absence of any performance deficits. Wagner et al. (2006) reported enhanced cortical (VLPFC and rostral ACC) activation in depressed patients performing a Stroop cognitive control task at normal levels. Harvey et al. (2005) suggested that the increases in cortical response they observed to their n-back challenge were caused by 'cortical inefficiency'. Their participants did not show behavioural deficits, so greater cortical activity could be a neural manifestation of the greater effort required to maintain normal performance. Other studies have provided support for this theory. Langenecker et al. (2007) found that successful response inhibition in depressed subjects was associated with enhanced cortical activation. Similarly, Walter et al. (2007) assessed patients' neural response to correct trials only in a working memory task and found increased DLPFC activation. This effect was not seen when incorrect trials were included in the analysis, suggesting that matched performance is associated with increased cortical response. Several other studies have also reported prefrontal hyperactivity associated with intact performance on working memory tasks (Matsuo et al., 2007; Walsh et al., 2007).

By contrast, where patients' performance is impaired, as in the Elliott et al. (1997a) study, reduced neuronal response is typically observed in cortical regions. Okada et al. (2003) found reduced activity in left VLPFC in depressed patients during a verbal fluency task on which they also performed poorly. Hugdahl et al. (2004) found impaired performance and decreased right inferior parietal activity in depressed participants during a mental arithmetic task (although medial prefrontal function was enhanced). It therefore seems that impaired performance is associated with reduced cortical function while normal performance can only be achieved through enhanced cortical function.

However, it is not always the case that normal executive performance is associated with increased cortical activation in depression. Barch et al. (2003) reported attenuated activity in depressed participants to both word and face versions of an n-back task in bilateral thalamus, right precentral gyrus and right parietal cortex despite no behavioural deficit being observed. It should be noted that differences were not observed in the prefrontal cortex and also that performance was at, or near, ceiling. It is possible that hyperfrontality associated with intact performance is only observed for more challenging versions of tasks. These discrepancies highlight the importance of careful characterisation of the relationship between task difficulty, performance and brain response. It is also possible that differences in patient demographics, severity of illness and medication status may contribute to the observed discrepancies and these issues should be addressed systematically in future research.

\section{Memory}

Memory problems are one of the most common cognitive symptoms reported by depressed patients; however there are very few fMRI studies of non-emotional memory in depression. Bremner et al. (2004) found that conceptual memory encoding of a neutral paragraph resulted in reduced hippocampal, amygdala and ACC activation and increased right frontal gyri activation in depressed subjects. By contrast, Werner et al. (2009) reported increased parahippohampal activity at encoding and decreased activity in frontal and parietal regions during both encoding and retrieval. Performance was unimpaired. The discrepancy may reflect differences in patient characteristics, in particular their medication status (Bremner's patients were unmedicated; Werner's were mostly receiving antidepressants). Task differences are also a factor, with the studies assessing conceptual prose memory compared with associative memory for picture-word pairs. However it is also important to note that structural hippocampal pathology has been widely discussed in some depression subtypes and may represent an important confound in memory studies.

\section{EMOTIONAL COGNITIVE NEUROIMAGING IN CURRENT DEPRESSION}

Neural responses to emotional tasks in depressed patients have been more widely studied using fMRI than responses to non-emotional tasks. Studies in normal subjects have identified the amygdala as playing a key role in processing faces and facial emotions (Adolphs et al., 1994; Breiter et al., 1996; Morris et al., 1996; Haxby et al., 2000; Phan et al., 2002). Amygdala function has also been linked to enhanced memory for emotional material (Cahill et al., 1995; Canli et al., 2000) and general processing of emotional material (Costafreda et al., 2008). Emotional disturbance is at the core of depressive symptomatology, and cognitive disturbances interact with the affective tone of stimulus material. Thus, depressed patients show negative biases in attentional and memory processing and a tendency to interpret information negatively (Clark et al., 2009 for review). In recent years, there have been numerous neuroimaging studies focused on the function of the amygdala, and interconnected regions, in mediating these emotional processing disturbances.

\section{Face emotion processing}

Some brain imaging studies have found that depressed patients show greater amygdala response to emotional faces than controls (Sheline et al., 2001; Fu et al., 2004, 2008a; Surguladze et al., 2005), but others have failed to replicate this finding (Gotlib et al., 2005; Keedwell et al., 2005; Dannlowski et al., 2008; Lee et al., 2008) and one (Lawrence et al., 2004) reported reduced amygdala response. These studies also report group differences in a range of other brain areas including hippocampus, hippocampal gyrus, ACC, insula, fusiform gyrus, caudate, thalamus, ventral striatum and frontal, parietal and temporal regions. However, the exact regions reported for each emotion and the direction of the group differences vary. These inconsistencies may be attributable to differences in the paradigms used; for example some have used subliminal stimuli (Sheline et al., 2001; Dannlowski et al., 2008) while others have not. This highlights a possible role for standardised measures for exploring emotional function. As in non-emotional studies, differences in clinical characteristics may also influence findings; both severity of depression and medication status may be important variables.

\section{Emotional words and pictures}

Although faces are most widely used, there have also been studies using other emotional stimuli. Siegle et al. $(2002,2006,2007)$ used self-referential emotionally valenced words and found that 
amygdala response to negative words was both increased and sustained for significantly longer in depressed participants compared to controls. Kumari et al. (2003) used pictures and sentences paired to create either a positive, negative or neutral message. In response to negative stimuli, they found decreased ACC response in depressed patients. Decreased responses in left medial frontal gyrus/ ACC and hippocampus were also observed for positive stimuli. Similarly, Mitterschiffthaler et al. (2003) used positive and neutral pictures with anhedonic depressed patients and found reduced medial orbitofrontal cortex (OFC) responses to positive pictures in depressed patients. Interestingly, depressed participants had greater response to positive pictures in lateral OFC, an area typically associated with negative emotion and punishment (O'Doherty et al., 2001; Kringelbach, 2005). These findings suggest that depressive anhedonia may be mediated by altered balances in normal emotional processing systems. Different symptom profiles may be associated with differential responses to emotional stimuli, suggesting once again that it is important to carefully characterise patient groups to understand differences between studies.

\section{Emotional processing biases}

An important dimension of depressive symptomatology is the biasing effect of emotional valence on cognitive performance: patients are biased towards processing negative information. Dannlowski et al. (2007) found that bilateral amygdala response to masked negative face emotions correlated positively with a bias towards judging faces more negatively, an effect not seen for happy faces. More explicitly, Hamilton and Gotlib (2008) examined the neural basis of negative memory biases in depression. They reported that depressed participants displayed greater right amygdala activity in response to negative (but not positive) emotional pictures that they subsequently remembered. Memory for the negative pictures was also enhanced, suggesting that amygdala hyperactivity at encoding could be a basis for negatively biased memory. However, findings in this area are inconsistent; Roberson-Nay et al. (2006) also reported greater left amygdala activation in response to subsequentlyremembered emotional stimuli (faces), but this was seen for happy and neutral faces as well as negative ones.
Studies have also considered the neuronal basis of affective biases in the attentional domain. Mitterschiffthaler et al. (2008) used an emotional Stroop task and showed that behavioural bias towards negative stimuli in depressed participants was associated with enhanced ACC response. Similarly, Elliott et al. (2002) reported increased ACC response to sad targets in an affective gono go task in depressed patients, but reduced response to happy targets; the exact opposite of the pattern observed in healthy controls. See Figure 1.

Sad distracters were associated with enhanced OFC response in patients, an effect attributed to a failure to inhibit instinctive bias towards sad information. Wang et al. (2008) also reported enhanced inferior frontal response associated with reduced ability to disengage from sad distracters. Similarly, Dichter et al. (2009) observed greater activation in depressed patients in a number of prefrontal regions, including inferior, and orbitofrontal gyri and ACC, in response to neutral targets embedded in sad compared to neutral contexts, suggesting that relatively more prefrontal brain activation was required to disengage from sad images to respond to the target events. Matthews et al. (2009) suggested that inhibitory control abnormalities in depression may not be confined to the affective domain, observing abnormal inferior frontal and ACC function during a non-affective inhibitory control paradigm. However, they nevertheless argued that these inhibitory control deficits may be important determinants of negative processing biases in depression.

\section{Performance feedback}

Another area where emotional biases contribute to cognitive function in depression is in a proposed abnormal response to negative performance feedback (Elliott et al., 1997b; Murphy et al., 2003). Taylor-Tavares et al. (2008) examined the neural basis of this effect. They found that compared to controls, depressed participants failed to deactivate the right amygdala in response to negative feedback. The amygdala deactivation seen in controls may be a defence mechanism to allow them to continue with the task without being adversely affected by failure, a mechanism which may be reduced in depression. Steele et al. (2007) reported an attenuation of the
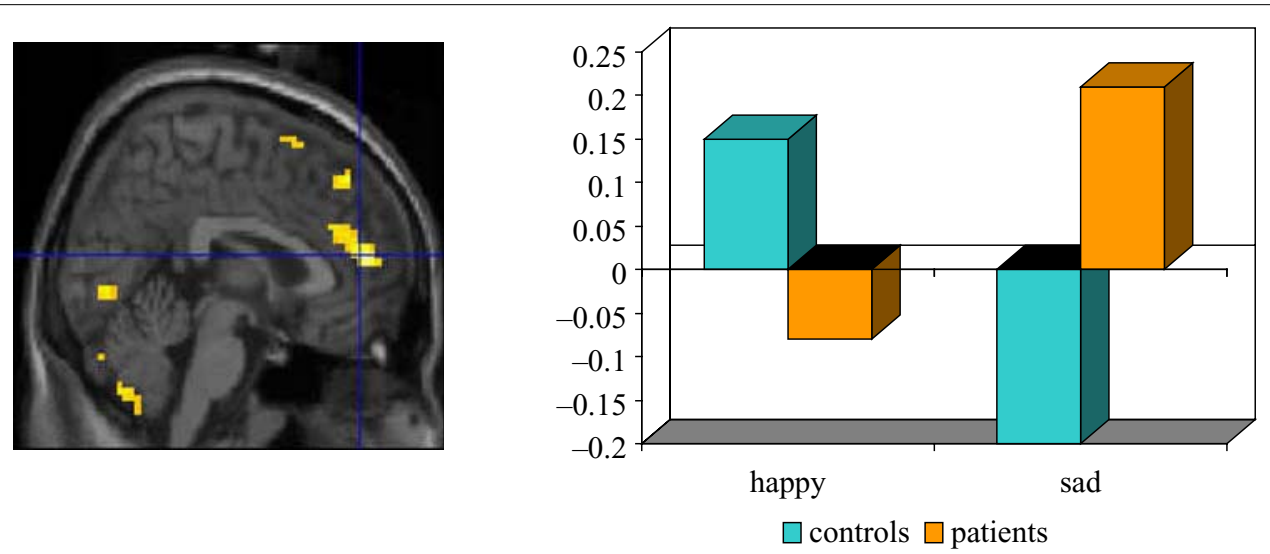

FIGURE 1 | Ventral ACC response to sad targets in depressed patients and happy targets in controls performing an affective go/nogo task. The left panel shows the focus of BOLD response and the right panel shows the adjusted BOLD response to happy and sad targets in patients and controls within this focus. Adapted from Elliott et al. (2002). 
normal ACC response to negative feedback in depressed patients. The ACC is closely connected with the amygdala and these findings, combined with those of Taylor-Tavares et al. (2008), may suggest that response to negative feedback in depression is mediated by a dysfunctional balance between the emotional functions of the amygdala and the cognitive control functions of the ACC.

\section{NETWORK MODELS OF DEPRESSION}

The literature reviewed above highlights the complexity inherent in understanding the functional neuroanatomy of impaired cognition in depression, with various discrepancies and interpretational issues still unresolved. Further research in well-characterised samples will be needed to resolve these issues fully. However, some general trends emerge. Specifically, there is strong support for the theory that normally performing depressed patients show hyperresponse within lateral frontal regions, while impaired performance is usually accompanied by hypo-response. These results are typically interpreted as representing inefficient cognitive processing within prefrontal regions. Negative emotional information is more salient to patients with depression compared to controls. Functional responses of limbic regions, particularly the amgydala, have been associated with biases observed in depressed patients towards negative information (faces, words, pictures, feedback). The ACC plays a role in both cognitive and emotional processing and the interface between them. Many activation studies in depression suggest abnormal ACC response but the nature of this abnormality (hypofunction vs. hyperfunction) varies widely depending on the cognitive challenge.

In general terms, disrupted cortico-limbic circuits, with a key modulating function for the ACC may explain both emotional biases and cognitive deficits in depression, as suggested in an influential model proposed by Mayberg (1997) and Mayberg et al. (1999). See Figure 2.

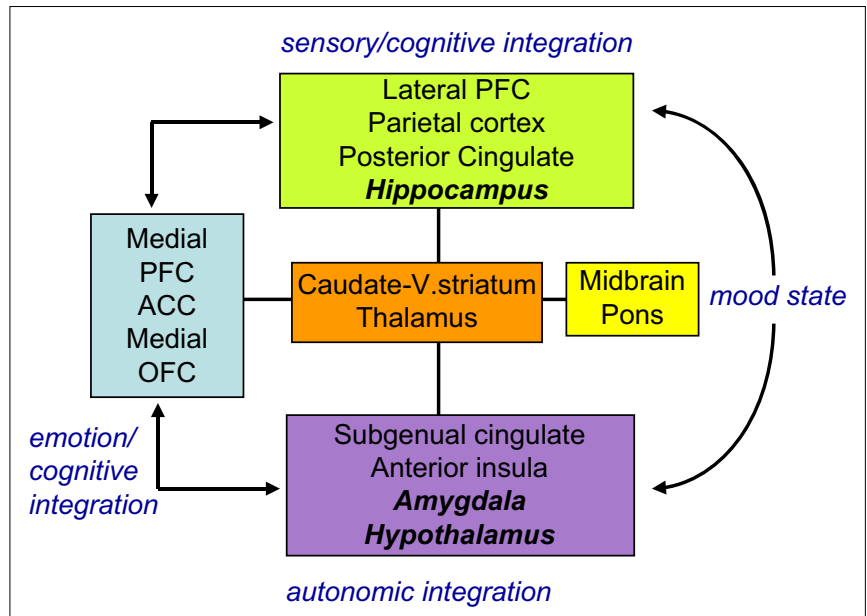

FIGURE 2 | Limbic-cortical dysregulation model. Interconnected regions are grouped into four 'compartments' relating to particular cognitive/ behavioural functions. Within compartments there may be a segregation between areas (shown in plain and italic text) showing an inverse relationship on different imaging paradigms. These interactions are dysfunctional in depression and modulated by successful treatment. Adapted from Mayberg (1997) and Mayberg et al. (1999).
This model has been developed and refined in recent years and shown to have diagnostic value and a role in predicting treatment response (Mayberg, 2002, 2003). Network models of depression, directly generated from functional neuroimaging results, can be tested explicitly using new connectivity analysis techniques.

\section{CONNECTIVITY ANALYSES}

The consensus view holds that depression is mediated by disruptions within networks of interconnected brain regions, rather than depending on abnormal function within a particular region. While the results discussed above are certainly supportive of this view, more recent approaches have addressed connectivity directly. One approach is to explore the extent to which response in different brain regions is intercorrelated. For example, Hamilton and Gotlib (2008) demonstrated that right amygdala response to remembered negative pictures was more strongly correlated with ipsilateral hippocampus and caudate-putamen response in depressed subjects. A similar approach is to consider functional coupling between brain regions in response to particular cognitive challenges. Several studies have explored functional coupling of the amygdala and prefrontal regions (Johnstone et al., 2007; Siegle et al., 2007; Chen et al., 2008; Matthews et al., 2008). Matthews et al. (2008) reported reduced functional coupling of the amygdala and supragenual ACC during emotion processing associated with increasing severity of depression. Similarly, Chen et al. (2008) reported increased functional coupling of amygdala and ACC during face emotion processing following antidepressant treatment (i.e. as symptom severity was reduced). Siegle et al. (2007) observed that depressed patients showed enhanced amygdala response during emotional tasks, reduced DLPFC response during executive tasks and a decreased functional coupling between amygdala and DLPFC, highlighting the importance of the cognitive challenge in exploring connectivity.

A more comprehensive approach to connectivity is to use techniques such as structural equation modelling and dynamic causal modelling to explore changes in a pre-specified network of regions. Using this approach, Schlosser et al. (2008) assessed connectivity associated with a cognitive control task (Stroop) and observed enhanced task-related input from the dorsal to rostral ACC in subjects with depression, perhaps signalling a failure to downregulate rostral ACC function in an inhibitory control context. Recent unpublished work from our team (Goulden et al., 2009) suggests that there are also abnormalities when dynamic causal modelling is applied to remitted depressed patients performing a face processing task, as shown in Figure 3.

In the most explicit test of a disrupted connectivity model to date, Seminowicz et al. (2004) applied structural equation modelling in a path modelling meta-analysis. This approach tested the specificity and reliability of Mayberg's reciprocal connectivity model (Figure 2) across different studies. The approach suggested different patterns of disrupted connectivity in different subgroups of patients, in particular identifying differences between responders and non-responders to treatment. Thus for connectivity studies, as for regional response studies, it is important to fully characterise patient groups in order to compare different results.

The studies reviewed briefly in this section suggest that connectivity approaches to analysing functional imaging data from depressed patients represent an important step forward in understanding 


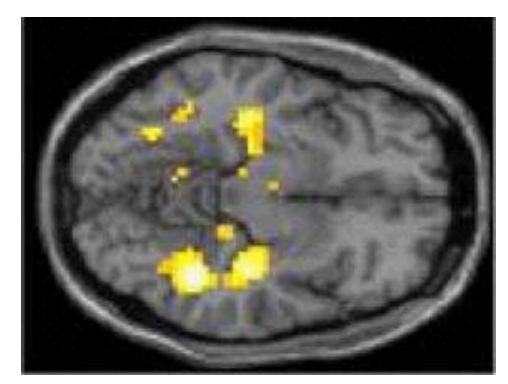

FIGURE 3 | Sad face processing in remitted depressed patients. The left panel shows attenuated bilateral hippocampus and fusiform signal in patients compared to controls. The right panel shows altered connectivity in patients obtained via dynamic causal modelling. The model tested was a right hemisphere model comprising primary visual cortex (V1), fusiform gyrus (FG),

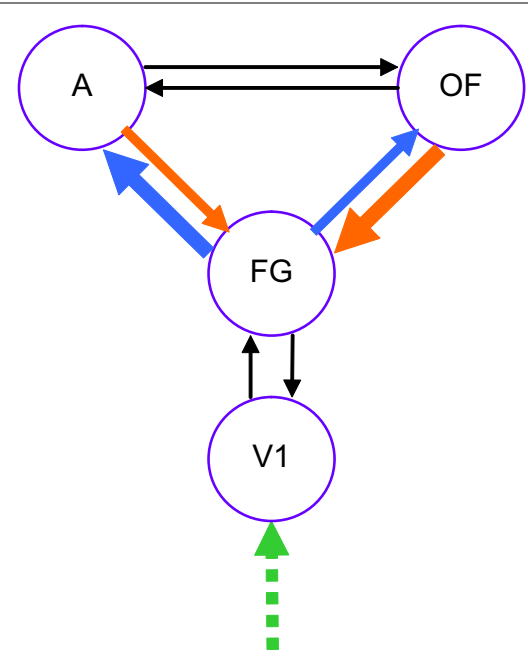

Input: sad faces

Amygdala (A) and orbitofrontal cortex (OFC). Connections shown in blue are stronger in patients (significant at $p<0.05$ corrected for boldest arrow) while those in orange are weaker in patients (significant at $p<0.05$ corrected for boldest arrow). Data acquired at the Wellcome Trust Clinical Research Facility, Manchester (Thomas et al., submitted; Goulden et al., 2009). the disorder. Effective connectivity between limbic and prefrontal regions is disrupted in depression and the nature of this disruption depends on the cognitive challenge presented. The Seminowicz et al. (2004) study also emphasises that it is important to study different subgroups and to consider treatment response.

\section{CLINICAL IMPLICATIONS OF COGNITIVE IMAGING}

Many of the studies discussed in this review have suggested important relationships between clinical features and functional response to cognitive challenges. Some studies have reported correlations with severity; others have reported changes in response to treatment. Discrepancies between studies may also point to distinct functional response profiles in different subtypes of depression. While a detailed discussion of these issues is beyond our scope, we will highlight two areas that illustrate the potential of neuroimaging cognition to inform clinical approaches to depression.

\section{REMITTED DEPRESSION STUDIES AS A MODEL FOR TRAIT EFFECTS}

Studying the neuronal basis of cognitive function in remitted depression provides a model for considering which abnormalities may represent trait effects or vulnerability markers. Despite the growing literature highlighting persistent cognitive deficits and biases in remitted depression, few studies have investigated the neural correlates of these cognitive findings. In a cross-sectional study, Takami et al. (2007) found ACC activity to a verbal fluency task was attenuated in elderly remitted depressives who had experienced multiple previous episodes, though not in those who had experienced only a single episode. By contrast, Hugdahl et al. (2007) used a longitudinal design to show that with remission, inferior frontal gyrus and the superior and inferior parietal lobule activity to a mental arithmetic task normalized in recurrent depressive participants.
Subtle emotional biases may remain in remitted depression, even when behaviour has normalised, and functional imaging provides a method for assessing these biases. Liotti et al. (2002) demonstrated that induced sad mood in remitted patients, but not controls, was associated with decreased function in ventromedial prefrontal regions and anterior thalamus, while VLPFC showed increases. Gemar et al. (2007) found a similar pattern of results in unmedicated remitted participants, indicating that these results are not due to residual medication effects. Combining mood induction and cognitive challenge, Ramel et al. (2007) reported that amygdala response to negative words during induced sad mood predicted subsequent recall in remitted participants.

There is also neuroimaging evidence for persistent abnormalities in depression in the absence of an induced depressed mood. Neumeister et al. (2006) demonstrated enhanced amygdala activation and reduced ventral striatum activation in remitted participants viewing sad faces (compared to neutral). In a recent study we found remitted participants to have increased neural responses to happy faces and reduced response to sad and fearful faces, in contrast to the opposite pattern more commonly described in current depression. We suggest that this may represent a maintenance mechanism for remission with participants suppressing the negative emotional biases associated with depressed mood (Thomas et al., submitted).

Using remission as a model for trait vulnerability is not without interpretational problems. Most obviously, it is unclear whether abnormalities in remitted patients represent genuine vulnerability traits, or 'scarring' from prior depressed episodes. One approach to address this confound is to consider never-depressed patients who may be vulnerable to developing the disorder. For example, Chan et al. (2009) have reported enhanced response to fearful faces in highly neurotic subjects considered at risk for depression. Similarly, 
Mannie et al. (2008) have reported impaired ACC response to emotional stimuli in young people with a strong family history of depression and therefore at risk themselves. However, these studies face the problem that relatively few of the subjects will go on to develop depression, and only long-term follow up can determine whether a specific pattern of response characterises these individuals. If imaging cognition could reliably identify individuals most vulnerable to depression, it may be possible to develop targeted intervention strategies for at-risk people that prevent development of depressive symptoms.

\section{TREATMENT EFFECTS ON COGNITION}

Serotonergic antidepressants have been associated with altered neuronal response to cognitive challenge in normal subjects. For example, Del-Ben et al. (2005) reported reduced limbic responses to negative faces after acute citalopram. Harmer et al. (2006) and Norbury et al. (2007) also reported reduced limbic response to negative faces after subacute doses of citalopram and reboxetine respectively. Similar findings have been reported in studies of antidepressant treatment in depressed patients. Studies have shown that participants who respond to treatment also have normalised (i.e. reduced) neural activity to emotional faces when retested (e.g. Sheline et al., 2001; Fu et al., 2004, 2007, 2008a). Neuronal correlates of response to psychological interventions have also been assessed (e.g. Roffman et al., 2005; Linden, 2006; Fu et al., $2008 b)$. Changes are reported in the neuronal correlates of affective processing following cognitive behavioural therapy or interpersonal therapy. Thus, different forms of antidepressant therapy directly impact abnormal functional responses, particularly in the emotional domain.

Functional imaging of cognitive challenge has also proved a potential predictor of treatment response, which has important implications for clinical practice (Ressler and Mayberg, 2007). Fu et al. (2008b) and Davidson et al. (2003) have reported that subjects who showed greatest ACC response to negative emotional stimuli at baseline responded best to antidepressant treatment. Meanwhile, Siegle et al. (2006) found that subjects with low subgenual ACC response and high amygdala response to emotional stimuli responded best to cognitive behavioural therapy.

While considerable further research is needed to explore relationships between treatment response and cognitive imaging abnormalities, these studies suggest an exciting role for brain imaging measures in the development of new algorithms for diagnosis and management of depressed patients (Mayberg, 2007). It may be possible to identify cognitive neuroimaging biomarkers that can predict which patients are likely to respond to a particular intervention (or which intervention is most likely to work for a particular patient). It may also be possible to identify which patients are more likely to relapse after treatment.

\section{FUTURE DIRECTIONS}

In this review, we have discussed a number of inconsistencies in the literature on imaging cognition in depression. These discrepancies highlight a need for more comprehensive reporting in some imaging studies, in relation to both clinical features of the sample and characterisation of the cognitive challenges used. For example, medication status of patients, duration of illness, number of previous episodes and any co-morbidity may all contribute to differences between studies. Drawing comparisons between studies and explaining apparently conflicting results critically depends on clear characterisation of the patients studied. Similarly, it is important to carefully characterise controls and match them as closely as possible to the patient sample; for example, it is relatively common for academic researchers to recruit controls among university staff and students, who may not represent a typical population sample. Furthermore, it is important for studies to relate BOLD response as closely as possible to cognitive performance. Directly relating regional responses to performance levels and considering responses to correct and incorrect trials separately, for example, may allow for a clearer understanding of the relationship between neuronal function and cognition in this subtly affected patient group. Thus, future research in this area should report subject characteristics and relationships between BOLD response and performance as comprehensively as possible to facilitate cross-study comparisons.

Although our review has largely focused on BOLD contrast fMRI, other imaging modalities have also been used to study cognitive function in depression. For example, magnetoencephalograpy (MEG) and electroencephalography (EEG) have been used and provide greater temporal resolution than fMRI, which may be important in studying processes like emotional arousal or inhibition (McNeely et al., 2008; Moratti et al., 2008). It is also clear that combining this approach with fMRI may help explain some of the discrepancies in the literature. For example, Wacker et al. (2009) combined fMRI and resting EEG to assess responses in reward systems associated with anhedonia. This multimodal approach showed that anhedonia was associated with reduced response to reward (fMRI) but also increased resting state activity in the nucleus accumbens (EEG). These results suggest that multimodal imaging represents an important future research direction.

Our review also highlights theoretical directions for future research, to test specific hypotheses arising from the existing literature. As discussed above, there is something of a dearth of nonemotional cognitive imaging. Future studies are needed to explore the relationship between performance and prefrontal function, systematically varying task difficulty to determine whether this is a critical determinant of hyper- vs. hypo-frontality. Furthermore, there is a need for a fuller exploration of the neural correlates of memory dysfunction, given that this is one of the commonest cognitive symptoms reported by patients. Memory dysfunction is reported most frequently in elderly patients with depression, and functional imaging studies are needed to relate this structural pathology in the hippocampus and to compare memory problems in late life depression with those observed in other conditions (mild cognitive impairment or early Alzheimer's disease).

Although the neuronal basis of emotional abnormalities has been studied more widely, further research is needed to relate these abnormalities more closely to clinical symptoms. Many studies have used rather heterogeneous clinical samples and future studies could compare subtypes of patients with distinct patterns of symptoms and with distinct aetiological features. Longitudinal studies in this area will also be critical in understanding how emotional abnormalities relate to response to different treatments and long-term 
prognosis. For both non-emotional and emotional cognitive neuroimaging, future research should exploit modern connectivity techniques to test network dysfunction hypotheses of depression.

Finally, brain imaging offers a powerful approach to exploring how genetic and environmental factors interact in conferring vulnerability and resilience to depression and large, systematic studies are needed in this area. As discussed above, this would provide the background for identifying neuroimaging biomarkers of both depression vulnerability and effective treatment, thus realising the potential of functional imaging in diagnosis and management.

\section{CONCLUSIONS}

In this review we have briefly summarised recent neuroimaging studies of non-emotional and emotional cognition in depression. These studies suggest that impaired executive function in depression is associated with inefficient cortical (and particularly

\section{REFERENCES}

Adolphs, R., Tranel, D., Damasio, H., and Damasio, A. (1994). Impaired recognition of emotion in facial expressions following bilateral damage to the human amygdala. Nature 372, 669-672.

American Psychiatric Association (1994). Diagnostic and Statistical Manual of Mental Disorders, 4th edn. Washington, DC, APA.

Barch,D.M.,Sheline,Y.I.,Csernansky, J. G., and Snyder, A. Z. (2003). Working memory and prefrontal cortex dysfunction: specificity to schizophrenia compared with major depression. Biol. Psychiatry 53, 376-384.

Breiter, H. C., Etcoff, N. L., Whalen, P. J., Kennedy, W. A., Rauch, S. L., Buckner, R. L., Strauss, M. M., Hyman, S. E., and Rosen, B. R. (1996). Response and habituation of the human amygdala during visual processing of facial expression. Neuron 17, 875-887.

Bremner, J. D., Vythilingam, M., Vermetten, E., Vaccarino, V., and Charney, D. S. (2004). Deficits in hippocampal and anterior cingulate functioning during verbal declarative memory encoding in midlife major depression. Am. J. Psychiatry 161, 637-645.

Cahill, L., Babinsky, R., Markowitsch, H. J., and Mcgaugh, J. L. (1995). The amygdala and emotional memory. Nature 377, 295-296.

Canli, T., Zhao, Z., Brewer, J., Gabrieli,J. D. E., and Cahill, L. (2000). Event-related activation in the human amygdala associates with later memory for individual emotional experience. J. Neurosci. 20, RC99(1-5).

Chan, S. W. Y., Norbury, R., Goodwin, G. M., and Harmer, C. J. (2009). Risk for depression and neural responses to fearful facial expressions of emotion. Br. J. Psychiatry 194,

Chen, C. H., Suckling, J., Ooi, C., Fu, C. H., Williams, S. C., Walsh, N. D., Mitterschiffthaler, M. T., Pich, E. M., Bullmore, E. (2008). Functional coupling of the amygdala in depressed patients treated with antidepressant medication. Neuropsychopharmacology 33, 1909-1918.

Clark, L., Chamberlain, S. R., and Sahakian, B. J. (2009). Neurocognitive mechanisms in depression: implications for treatment. Annu. Rev. Neurosci. 32, 57-74.

Costafreda, S. G., Brammer, M. J., David, A. S., and Fu, C. H. Y. (2008). Predictors of amygdala activation during the processing of emotional stimuli: a meta-analysis of 385 PET and fMRI studies. Brain Res. Rev. 58, 57-70.

Dannlowski, U., Ohrmann, P., Bauer, J., Deckert, J., Hohoff, C., Kugel, H., Arolt, V., Heindel, W., Kersting, A., Baune, B. T., and Suslow, T. (2008). 5-HTTLPR biases amygdala activity in response to masked facial expressions in major depression. Neuropsychopharmacology 33, 418-424.

Dannlowski, U., Ohrmann, P., Bauer, J., Kugel, H., Arolt, V., Heindel, W., and Suslow, T. (2007). Amygdala reactivity to masked negative faces is associated with automatic judgmental bias in major depression: a $3 \mathrm{~T}$ fMR1 study. J. Psychiatry Neurosci. 32, 423-429.

Davidson, R. J., Irwin, W., Anderle, M. J., and Kalin, N. H. (2003). The neural substrates of affective processing in depressed patients treated with venlafaxine. Am. J. Psychiatry 160, 64-75.

Del-Ben, C. M., Deakin, J. F., McKie, S., Delvai, N.A., Williams, S. R., Elliott, R., Dolan, M., and Anderson, I. M. (2005). 139-145.

lateral prefrontal) processing. Normal levels of performance can sometimes be achieved through recruitment of additional processing resources reflected in prefrontal hyperfunction. However, when normal performance cannot be achieved, prefrontal function is reduced. Emotional processing, particularly of negatively valenced material, is facilitated in depression and this is mediated by enhanced function of limbic regions, including the amygdala. The ACC plays a critical but complex role in depression, mediating the balance between limbic and cortical function in a context dependent manner. Models of cortico-limbic dysfunction (notably that of Mayberg, 1997; Mayberg et al., 1999) can explain this pattern of results, and modern connectivity techniques provide a means to test these models explicitly. Recent studies have started to identify the neurocognitive signature of vulnerability to depression and to assess and predict response to different treatment options. Neurocognitive assessment of depression using functional imaging therefore has exciting implications for diagnosis and treatment.

The effect of citalopram pretreatment on neuronal responses to neuropsychological tasks in normal volunteers: an fMRI study. Neuropsychopharmacology 30, 1724-1734.

Dichter, G.S.,Felder,J.N., and Smoski, M. J. (2009).Affective context interferes with cognitive control in unipolar depression: an fMRI investigation. J. Affect. Disord. 114, 131-142.

Elliott, R., Baker, S. C., Rogers, R. D., O'Leary, D.A., Paykel, E. S., Frith, C. D., Dolan, R. J., and Sahakian, B. J. (1997a) Prefrontal dysfunction in depressed patients performing a complex planning task: a study using positron emission tomography. Psychol. Med. 27, 931-942.

Elliott, R., Sahakian, B. J., Herrod, J. J., Robbins, T. W., and Paykel, E. S. (1997b). Abnormal response to negative feedback in unipolar depression: evidence for a diagnosis specific impairment. J. Neurol. Neurosurg. Psychiatr. 63, 74-82.

Elliott, R., Rubinsztein, J.S., Sahakian, B. J., and Dolan, R. J. (2002). The neural basis of mood-congruent processing biases in depression. Arch. Gen. Psychiatry 59, 597-604.

Fitzgerald, P. B., Oxley, T. J., Laird, A. R., Kulkarni, J., Egan, G. F., and Daskalakis, Z. J. (2006). An analysis of functional neuroimaging studies of dorsolateral prefrontal cortical activity in depression. Psychiatry Res. Neuroimaging 148, 33-45.

Fitzgerald, P. B., Srithiran, A., Benitez, J., Daskalakis, Z. Z., Oxley, T. J., Kulkarni, J., and Egan, G. F. (2008). An fMRI study of prefrontal brain activation during multiple tasks in patients with major depressive disorder. Hum. Brain Mapp. 29, 490-501.

Fu, C.H., Mourao-Miranda, J., Costafreda, S. G., Khanna, A., Marquand, A. F.,
Williams, S. C., and Brammer, M. J. (2008a). Pattern classification of sad facial processing: toward the development of neurobiological markers in depression. Biol. Psychiatry 63, 656-662.

Fu, C. H., Williams, S. C., Cleare, A. J., Scott, J., Mitterschiffthaler, M. T., Walsh, N. D., Donaldson, C., Suckling, J., Andrew, C., Steiner, H., and Murray, R. M. (2008b). Neural responses to sad facial expressions in major depression following cognitive behavioral therapy. Biol. Psychiatry 64, 505-512.

Fu, C. H., Williams, S. C., Brammer, M. J., Suckling, J., Kim, J., Cleare, A. J., Walsh, N. D., Mitterschiffthaler, M. T., Andrew, C. M., Pich, E. M., and Bullmore, E. T. (2007). Neural responses to happy facial expressions in major depression following antidepressant treatment. Am. J. Psychiatry 164, 599-607.

Fu, C. H., Williams, S. C., Cleare, A. J., Brammer, M. J., Walsh, N. D., Kim, J., Andrew, C. M., Pich, E. M., Williams, P. M., Reed, L. J., Mitterschiffthaler, M. T., Suckling, J., and Bullmore,E.T.(2004).Attenuation of the neural response to sad faces in major depression by antidepressant treatment - a prospective, eventrelated functional magnetic resonance imaging study. Arch. Gen. Psychiatry 61, 877-889.

Gemar, M. C., Segal, Z. V., Mayberg, H. S., Goldapple, K., and Carney, C. (2007) Changes in regional cerebral blood flow following mood challenge in drug-free, remitted patients with polar depression. Depress. Anxiety 24, 597-601.

Gotlib, I. H., Sivers, H., Gabrieli, J. D., Whitfield-Gabrieli, S., Goldin, P., Minor, K. L., and Canli, T. (2005). 
Subgenual anterior cingulate activation to valenced emotional stimuli in major depression. Neuroreport 16, 1731-1734.

Goulden, N., McKie, S., Pegg, E., Downey, D., Elliott, R., Williams, S. R., Anderson, I. M., and Deakin, J. F. W. (2009). Effective connectivity analysis of emotion processing in remitted depression. In Proceedings of the 17th Annual Meeting of ISMRM, Honolulu, pp. 3361.

Haldane, M., and Frangou, S. (2006). Functional neuroimaging studies in mood disorders. ActaNeuropsychiatrica 18, 88-99.

Hamilton, J. P., and Gotlib, I. H. (2008). Neural substrates of increased memory sensitivity for negative stimuli in major depression. Biol. Psychiatry 63, 1155-1162.

Harmer, C. J., Mackay, C. E., Reid, C. B., Cowen, P. J., and Goodwin, G. M. (2006).Antidepressant drug treatment modifies the neural processing of nonconscious threat cues. Biol. Psychiatry 59, 816-820.

Harvey, P. O., Fossati, P., Pochon, J. B., Levy, R., Lebastard, G., Lehéricy, S., Allilaire, J. F., and Dubois, B. (2005). Cognitive control and brain resources in major depression: an fMRI study using the n-back task. Neuroimage 26, 860-869.

Haxby, J. V., Hoffman, E. A., and Gobbini, M.I. (2000). The distributed human neural system for face perception. Trends Cogn. Sci. 4, 223-233.

Hugdahl, K., Rund, B. R., Lund, A., Asbjørnsen, A., Egeland, J., Ersland, L., Landrø, N. I., Roness, A., Stordal, K. I., Sundet, K., and Thomsen, T. (2004). Brain activation measured with fMRI during a mental arithmetic task in schizophrenia and major depression. Am. J. Psychiatry 161, 286-293.

Hugdahl, K., Specht, K., Biringer, E., Weis, S., Elliott, R., Hammar, A., Ersland, L., and Lund, A. (2007). Increased parietal and frontal activation after remission from recurrent major depression: a repeated fMRI study. Cognit. Ther. Res. 31, 147-160.

Johnstone, T., van Reekum, C. M., Urry, H. L., Kalin, N. H., and Davidson, R. J. (2007). Failure to regulate: counterproductive recruitment of top-down prefrontal-subcortical circuitry in major depression. J. Neurosci. 27, 8877-8884.

Keedwell, P. A., Andrew, C., Williams, S. C. R., Brammer, M. J., and Phillips, M. L. (2005). A double dissociation of ventromedial prefrontal cortical responses to sad and happy stimuli in depressed and healthy individuals. Biol. Psychiatry 58, 495-503.

Kringelbach, M. L. (2005). The human orbitofrontal cortex: linking reward to hedonic experience. Nat. Rev. Neurosci. 6, 691-702.

Kumari, V., Mitterschiffthaler, M. T., Teasdale,J.D.,Malhi,G.S.,Brown, R. G., Giampietro, V., Brammer, M. J., Poon, L., Simmons, A., Williams, S. C., Checkley, S.A., and Sharma, T. (2003). Neural abnormalities during cognitive generation of affect in treatmentresistant depression. Biol. Psychiatry 54, 777-791.

Langenecker, S. A., Kennedy, S. E., Guidotti, L. M., Briceno, E. M., Own, L. S., Hooven, T., Young, E. A., Akil, H., Noll, D. C., and Zubieta, J. K. (2007). Frontal and limbic activation during inhibitory control predicts treatment response in major depressive disorder. Biol. Psychiatry 62, 1272-1280.

Lawrence, N. S., Williams, A. M., Surguladze, S., Giampietro, V., Brammer,M.J.,Andrew,C., Frangou, S., Ecker, C., and Phillips, M. L. (2004). Subcortical and ventral prefrontal cortical neural responses to facial expressions distinguish patients with bipolar disorder and major depression. Biol. Psychiatry 55, 578-587.

Lee, B. T., Seok, J. H., Lee, B. C., Cho, S. W., Yoon, B. J., Lee, K. U., Chae, J. H., Choi, I. G., and Ham, B. J. (2008). Neural correlates of affective processing in response to sad and angry facial stimuli in patients with major depressive disorder. Prog. Neuropsychopharmacol. Biol. Psychiatry 32, 778-785.

Linden, D.E. (2006). How psychotherapy changes the brain - the contribution of functional neuroimaging. Mol. Psychiatry 11, 528-538.

Liotti, M., Mayberg, H. S., McGinnis, S., Brannan, S. L., and Jerabek, P. (2002). Unmasking disease-specific cerebral blood flow abnormalities: mood challenge in patients with remitted unipolar depression. Am. J. Psychiatry 159, 1830-1840.

Mannie, Z. N., Norbury, R., Murphy, S. E., Inkster, B., Harmer, C. J., and Cowen, P. J. (2008). Affective modulation of anterior cingulate cortex in young people at increased familial risk of depression. Br. J. Psychiatry 192, 356-361.

Matsuo, K., Glahn, D. C., Peluso, M. A., Hatch, J. P., Monkul, E. S., Najt, P., Sanches, M., Zamarripa, F., Li, J., Lancaster, J. L., Fox, P. T., Gao, J. H., and Soares, J. C. (2007). Prefrontal hyperactivation during working memory task in untreated individuals with major depressive disorder. Mol. Psychiatry 12, 158-166.

Matthews, S., Simmons, A., Strigo, I., Gianaros, P., Yang, T., and Paulus, M. (2009). Inhibition-related activity in subgenual cingulate is associated with symptom severity in major depression. Psychiatry Res. Neuroimaging 172, 1-6.

Matthews,S.C.,Strigo,I.A.,Simmons, A. N., Yang, T. T., and Paulus, M. P. (2008). Decreased functional coupling of the amygdala and supragenual cingulate is related to increased depression in unmedicated individuals with current major depressive disorder. J. Affect. Disord. 111, 13-20.

Mayberg, H. S. (1997). Limbic-cortical dysregulation: a proposed model of depression. J. Neuropsychiatry Clin. Neurosci. 9, 471-481.

Mayberg, H. S. (2002). Modulating dysfunctional limbic-cortical circuits in depression: targets of antidepressant treatments. Semin. Clin. Neuropsychiatry 7, 255-268.

Mayberg, H. S. (2003). Modulating dysfunctional limbic-cortical circuits in depression: towards development of brain-based algorithms for diagnosis and optimised treatment. Br. Med. Bull. 65, 193-210.

Mayberg, H. S. (2007). Defining the neural circuitry of depression: toward a new nosology with therapeutic implications. Biol. Psychiatry 61, 729-730.

Mayberg, H. S., Liotti, M., Brannan, S. K., McGinnis, S., Mahurin, R. K., Jerabek, P. A., Silva, J. A., Tekell, J. L., Martin, C. C., Lancaster, J. L., and Fox, P. T. (1999). Reciprocal limbiccortical function and negative mood: converging PET findings in depression and normal sadness. Am. J. Psychiatry 156, 675-682.

McNeely, H. E., Lau, M. A., Christensen, B. K., and Alain, C. (2008). Neurophysiological evidence of cognitive inhibition anomalies in persons with major depressive disorder. Clin. Neurophysiol. 119, 1578-1589.

Mitterschiffthaler, M. T., Kumari, V., Malhi, G. S., Brown, R. G., Giampietro, V. P., Brammer, M. J., Suckling, J., Poon, L., Simmons, A., Andrew, C., and Sharma, T. (2003). Neural response to pleasant stimuli in anhedonia: an fMRI study. Neuroreport 14, 177-182.

Mitterschiffthaler, M. T., Williams, S. C., Walsh, N. D., Cleare, A. J., Donaldson, C., Scott, J., and Fu, C. H. (2008). Neural basis of the emotional Stroop interference effect in major depression. Psychol. Med. 38, 247-256.

Moratti, S., Rubio, G., Campo, P., Keil, A., and Ortiz, T. (2008). Hypofunction of right temporoparietal cortex during emotional arousal in depression. Arch. Gen. Psychiatry 65, 532-41.

Morris, J. S., Frith, C. D., Perrett, D. I., Rowland,D., Young,A.W., Calder, A. J., and Dolan, R. J. (1996). A differential neural response in the human amygdala to fearful and happy facial expressions. Nature 383, 812-815.

Murphy, F. C., Michael,A., Robbins, T. W., and Sahakian, B. J. (2003). Neuropsychological impairment in patients with major depressive disorder: the effects of feedback on task performance. Psychol. Med. 33, 455-467.

Neumeister, A., Drevets, W. C., Belfer, I., Luckenbaugh, D. A., Henry, S., Bonne, O.,Herscovitch, P., Goldman, D., and Charney, D. S. (2006). Effects of alpha(2C)-adrenoreceptor gene polymorphism on neural responses to facial expressions in depression. Neuropsychopharmacology 31, 1750-1756.

Norbury, R., Mackay, C. E., Cowen, P. J., Goodwin, G. M., and Harmer, C. J. (2007). Short-term antidepressant treatment and facial processing functional magnetic resonance imaging study. Br. J. Psychiatry 190, 531-532.

O’Doherty, J., Kringelbach, M. L., Rolls, E. T.,Hornak, J., and Andrews, C. (2001). Abstract reward and punishment representations in the human orbitofrontal cortex. Nat. Neurosci. 4, 95-102.

Okada, G., Okamoto, Y., Morinobu, S., Yamawaki, S., and Yokota, N. (2003). Attenuated left prefrontal activation during a verbal fluency task in patients with depression. Neuropsychobiology 47, 21-26.

Phan, K. L., Wager, T., Taylor, S. F., and Liberzon, I. (2002). Functional neuroanatomy of emotion: a meta-analysis of emotion activation studies in PET and fMRI. Neuroimage 16, 331-348.

Ramel, W., Goldin, P. R., Eyler, L. T., Brown, G. G., Gotlib, I. H., and McQuaid, J. R. (2007). Amygdala reactivity and mood-congruent memory in individuals at risk for depressive relapse. Biol. Psychiatry 61, 231-239.

Ressler, K. J., and Mayberg, H. S. (2007). Targeting abnormal neural circuits in mood and anxiety disorders: from the laboratory to the clinic. Nat. Neurosci. 10, 1116-1124.

Roberson-Nay, R., McClure, E. B., Monk, C. S., Nelson, E. E., Guyer, A. E., Fromm, S. J., Charney, D. S., Leibenluft, E., Blair, J., Ernst, M., and Pine, D. S. (2006). Increased amygdala activity during successful memory encoding in adolescent major depressive disorder: an fMRI study. Biol. Psychiatry 60, 966-973.

Roffman, J. L., Marci, C. D., Glick, D. M., Dougherty, D. D., and Rauch, S. L. (2005). Neuroimaging and the functional neuroanatomy of psychotherapy. Psychol. Med. 35, 1385-98. 
Schlosser, R. G. M., Wagner, G., Koch, K., Dahnke, R., Reichenbach, J. R., and Sauer, H. (2008). Fronto-cingulate effective connectivity in major depression: a study with $\mathrm{PMRI}$ and dynamic causal modeling. Neuroimage 43, 645-655.

Seminowicz, D. A., Mayberg, H. S., McIntosh, A. R., Goldapple, K., Kennedy, S., Segal, Z., and Rafi-Tari, S. (2004). Limbic-frontal circuitry in major depression: a path modeling metanalysis. Neuroimage 22, 409-418.

Sheline, Y.I., Barch, D. M., Donnelly, J. M., Ollinger, J. M., Snyder, A. Z., and Mintun, M. A. (2001). Increased amygdala response to masked emotional faces in depressed subjects resolves with antidepressant treatment: an fMRI study. Biol. Psychiatry 50, 651-658.

Siegle, G. J., Carter, C. S., and Thase, M. E. (2006). Use of fMRI to predict recovery from unipolar depression with cognitive behavior therapy. Am. J. Psychiatry 163, 735-737.

Siegle, G. J., Steinhauer, S. R., Thase, M. E., Stenger, V.A., and Carter, C. S. (2002). Can't shake that feeling: assessment of sustained event-related fMRI amygdala activity in response to emotional information in depressed individuals. Biol. Psychiatry 51, 693-707.

Siegle, G. J., Thompson, W., Carter, C. S., Steinhauer, S. R., and Thase, M. E.
(2007). Increased amygdala and decreased dorsolateral prefrontal BOLD responses in unipolar depression: related and independent features. Biol. Psychiatry 61, 198-209.

Steele, J. D., Kumar, P., and Ebmeier, K. P. (2007). Blunted response to feedback information in depressive illness. Brain 130, 2367-2374.

Surguladze,S., Brammer,M.J., Keedwell, P., Giampietro, V., Young, A. W., Travis, M. J., Williams, S. C., and Phillips, M. L. (2005). A differential pattern of neural response toward sad versus happy facial expressions in major depressive disorder. Biol. Psychiatry 57, 201-209.

Takami, H., Okamoto, Y., Yamashita, H., Okada, G., and Yamawaki, S. (2007). Attenuated anterior cingulate activation during a verbal fluency task in elderly patients with a history of multiple-episode depression. Am. J. Geriatr. Psychiatry 15, 594-603.

Taylor-Tavares, J. V., Clark, L., Furey, M. L., Williams, G. B., Sahakian, B. J., and Drevets, W. C. (2008). Neural basis of abnormal response to negative feedback in unmedicated mood disorders. Neuroimage 42, 1118-1126.

Thomas, E. J., Elliott, R., McKie, S., Arnone, D., Downey, D., Juhasz, G., Deakin, J. F. W., and Anderson, I. M. Effect of past depression and rumination on neural response to emotional faces. Submitted.

Wacker, J., Dillon, D. G., and Pizzagalli, D. A. (2009). The role of the nucleus accumbens and rostral anterior cingulate cortex in anhedonia: integration of resting EEG, fMRI, and volumetric techniques. Neuroimage 46, 327-37.

Wagner, G., Sinsel, E., Sobanski, T., Köhler, S., Marinou, V., Mentzel, H. J., Sauer, H., and Schlösser, R. G. (2006). Cortical inefficiency in patients with unipolar depression: an event-related fMRI study with the Stroop task. Biol. Psychiatry 59, 958-965.

Walsh,N.D.,Williams,S.C.,Brammer, M. J. Bullmore, E. T., Kim, J., Suckling, J., Mitterschiffthaler, M. T., Cleare, A. J., Pich, E. M., Mehta, M.A., and Fu, C. H. (2007). A longitudinal functional magnetic resonance imaging study of verbal working memory in depression after antidepressant therapy. Biol. Psychiatry 62, 1236-1243.

Walter, H., Wolf, R. C., Spitzer, M., and Vasic, N. (2007). Increased left prefrontal activation in patients with unipolar depression: an event-related, parametric, performance-controlled fMRI study. J. Affect. Disord. 101, 175-185.

Wang, L., LaBar, K. S., Smoski, M., Rosenthal, M. Z., Dolcos, F. Lynch, T. R., Krishnan, R. R., and McCarthy, G. (2008). Prefrontal mechanisms for executive control over emotional distraction are altered in major depression. Psychiatry Res. Neuroimaging 163, 143-155.

Werner, N. S., Meindl, T., Materne, J., Engel, R. R., Huber, D., Riedel, M. Reiser, M., and Hennig-Fast, K. (2009). Functional MRI study of memoryrelated brain regions in patients with depressive disorder. J. Affect. Disord. (in press).

Conflict of Interest Statement: The authors declare that the research was conducted in the absence of any commercial or financial relationships that could be construed as a potential conflict of interest.

Received: 29 May 2009; paper pending published: 21 August 2009; accepted: 24 September 2009; published online: 09 October 2009.

Citation: Thomas EJ and Elliott R (2009) Brain imaging correlates of cognitive impairment in depression. Front. Hum. Neurosci. 3:30. doi: 10.3389/neuro.09.030.2009 Copyright $\odot 2009$ Thomas and Elliott. This is an open-access article subject to an exclusive license agreement between the authors and the Frontiers Research Foundation, which permits unrestricted use, distribution, and reproduction in any medium, provided the original authors and source are credited. 